\title{
Pour que les étudiants de FLS comprennent et participent à la francophonie canadienne
}

\author{
Laurence Valérie Thibault \\ Ithibau2@uottawa.ca \\ Marie-Claude Dansereau \\ Sylvie Lamoureux \\ UNIVERSITÉ D'OTTAWA
}

\begin{abstract}
Résumé
Le présent article est issu d'un atelier qui, à la lumière de résultats probants de deux recherches complémentaires sur les parcours pédagogiques de futurs enseignants de français langue seconde, présente deux réflexions de pratique d'enseignement du FLS en milieu universitaire. La recherche constate le manque de littératie culturelle et linguistique - notamment concernant la francophonie canadienne - des futurs enseignants de français parmi lesquels se trouvent des apprenants avancés de français langue seconde (FLS) (Lamoureux et Cohen, 2012; Moor et Lamoureux, 2012). Deux réflexions de pratique illustrent comment développer cette littératie dans un programme universitaire de FLS. La première traite du développement des compétences interculturelles (Byram, 2003) et présente des projets pédagogiques permettant aux étudiants de participer à la francophonie canadienne de manière autonome et éclairée. La deuxième porte sur l'importance d'accorder une valeur didactique à la compréhension des variétés locales de la langue pour mieux conscientiser les étudiants à la fonction symbolique de celle-ci (Cuq et Gruca, 2002) et les amener à communiquer avec les locuteurs natifs de manière plus autonome.
\end{abstract}

Mots-clés : apprentissage du français langue seconde, littératie socioculturelle et sociolinguistique

\section{Abstract}

This article is based on a second language teaching workshop drawing on the key findings of research into the academic pathways of future French as a second language (FSL) educators. The research reveals a lack of cultural and linguistic literacy reported by future FSL teachers - including advanced learners of FSL - in terms of Canada's francophone communities (Lamoureux and Cohen, 2012; Moor and Lamoureux, 2012). The two lines of thinking on pedagogical practice presented in this article demonstrate how these literacies can be developed in a university FSL program. The first pedagogical practice highlights the development of intercultural

\section{(C)CAHIERS DE L'ILOB Vol. 7, 2015 127-140}


competency (Byram, 2003) through pedagogical projects that enable students to actively and autonomously participate in the Canadian "francophonie". The second pedagogical practice demonstrates the importance of a didactic legitimization of students' comprehension of local language varieties of French and their symbolic function (Cuq and Gruca, 2002). This awareness enables students to communicate effectively with local native speakers of French.

Key words: French second language learning, sociocultural and sociolinguistic literacies

\section{Introduction}

Le présent article traite de littératie et d'autonomie chez les futurs enseignants de français et les apprenants avancés de français langue seconde (FLS). En réponse à des résultats de recherches empiriques qui soulignent le besoin de développer cette littératie et cette autonomie chez les apprenants avancés de FLS et les futurs enseignants de FLS, nous présentons deux pratiques de classe qui illustrent comment répondre à ce besoin. Ici, la littératie est comprise dans son sens large. Elle inclut les compétences à l'oral — notamment vis-à-vis des variétés régionales et des registres de langue - ainsi que les compétences (inter)culturelles relativement à la francophonie canadienne. L'autonomie des apprenants de FLS est envisagée comme un but à atteindre aux plans linguistique et culturel. Cette autonomie est d'autant plus vitale que les étudiants qui s'inscrivent aux cours de FLS ont des ambitions professionnelles : obtenir un emploi bilingue ou devenir enseignant de FLS en contexte minoritaire.

\section{Problématique contextualisée par les résultats de recherche}

En 2009, Salvatori et Macfarlane publient les résultats d'une recherche menée pour le compte de l'Association canadienne des professeurs de langue seconde (ACPLS). Leur rapport met en lumière les nombreux défis que vivent les enseignants de FLS au Canada, dont deux résultats retiennent notre attention. Les enseignants qui ont participé à l'étude indiquent ne pas suffisamment maîtriser les compétences langagières pour donner avec confiance les cours de FLS de la maternelle à la $12^{\mathrm{e}}$ année. De plus, peu d'enseignants indiquent avoir les compétences et les connaissances culturelles en français qu'ils estiment nécessaires pour un enseignement efficace en FLS.

Les résultats de cette recherche, présentés dans le cadre d'un cours de Didactique des langues secondes (DLS) à l'automne 2010, ont mis en lumière le questionnement d'étudiants en fin de parcours scolaire, à l'aube de leur transition à la formation initiale à l'enseignement, par rapport à l'apprentissage des compétences et des connaissances culturelles en français lors de leur cursus universitaire. Ce questionnement incite Lamoureux et Cohen (2012) à 
faire une analyse des parcours de formation postsecondaire en FLS offerts dans les universités de l'Ontario. Elles concluent que la grande majorité des parcours de formation de premier cycle et de formation initiale à l'enseignement offrent peu ou pas d'occasions aux étudiants de bonifier leurs connaissances ou compétences quant aux variétés de français (canadiennes et autres), ou aux référents culturels canadiens-français hors Québec, qu'ils soient historiques ou d'actualité.

Face à ces constats d'analyse de programme, Moor et Lamoureux (2012) entreprennent une étude de cas auprès d'étudiants maîtres, option didactique du FLS, en fin de parcours de formation initiale à l'enseignement à l'Université d'Ottawa. Leur intention est de cerner les perceptions d'étudiants maîtres quant à leur compétence et leur degré de confort à intégrer les référents culturels de la francophonie locale, provinciale, nationale et internationale à leur future pratique d'enseignement du FLS en Ontario. Moor, elle-même apprenante avancée du FLS et étudiante maître en didactique du FLS, a mené l'enquête sous la supervision de la professeure Lamoureux dans le cadre du programme d'initiation à la recherche pour les étudiants du premier cycle de l'Université d'Ottawa. Les résultats du groupe de discussion mené par Moor en mars 2012, où participaient cinq étudiants francophones et apprenants avancés du français recrutés dans les cours de didactiques du FLS à la formation initiale et à la Teacher Education à l'Université d'Ottawa, démontrent qu'aucun de ces étudiants ne participe régulièrement à l'espace francophone en Ontario. De plus, ces étudiants maitres de FLS, y compris les francophones, ne se sentent pas capables d'assumer le rôle de passeur ou médiateur culturel, qui est dorénavant intégré à l'enseignement du français langue seconde en Ontario.

Le nouveau cadre de référence pour l'enseignement du français langue seconde en Ontario (Ontario, 2013a) et le curriculum révisé du FLS en Ontario (Ontario, 2013b) mettent en lumière des attentes élevées pour les enseignants de FLS, actuels et futurs, et leurs élèves. Le cadre de référence (Ontario 2013a) précise les visées des programmes de FLS en Ontario, où l'élève pourra entre autres, communiquer avec des francophones en situations authentiques et participer à la culture et à l'espace francophones, alors que le personnel enseignant a la responsabilité d'augmenter les contacts et les partenariats avec les organismes francophones (Ontario, 2013a, pp. 16, 19) pour permettre ces deux visées. Puis, le curriculum révisé (Ontario, 2013b) souligne entre autres l'importance de l'enseignant comme modèle francophone, soit un passeur/médiateur des cultures francophones (p. 13); l'interdépendance entre la langue et la culture (p. 10); et la possibilité pour l'élève d'utiliser le français dans une variété de contextes sociaux, lui offrant ainsi les outils qui lui permettent de participer à l'espace francophone en Ontario, au Canada ou ailleurs (p. 11). 
Face aux constats des recherches de Salvatori et Macfarlane (2009), de Moor et Lamoureux (2012) et de Lamoureux et Cohen (2012), comment les étudiants, futurs enseignants de FLS, et les étudiants de FLS peuvent-ils s'approprier les connaissances et les compétences (inter)culturelles en lien avec le français d'ici, mais également avec des variétés locales de la langue, afin de pouvoir participer à l'espace francophone et d'être confiants dans leur travail, que ce soit à titre d'enseignant de FLS ou d'employé bilingue ? Dans les faits, Lamoureux et Cohen ont relevé deux cours optionnels de FLS à l'Université d'Ottawa qui permettent aux étudiants d'approfondir leurs connaissances du français et de la culture francophone d'ici, FLS 4775 Culture et communication en français langue seconde et FLS 4774 Compréhension du français d'ici.

Ces deux cours, que nous décrirons dans cet article et que nous qualifierons de pratiques réussies, sont offerts à des étudiants de niveau perfectionnement (soit le quatrième niveau sur quatre), équivalant à un niveau situé entre B2 et C1 du Cadre européen commun de référence pour les langues (CECRL, Conseil de l'Europe, 2001). La majorité de ces étudiants sont en troisième ou quatrième année d'université. Plusieurs sont inscrits à un programme de FLS ou de DLS et souhaitent devenir des enseignants. Ils proviennent de différents programmes (français de base, français enrichi, immersion) et leur connaissance de la francophonie varie énormément entre eux. Ces deux cours, enseignés exclusivement en français, illustrent bien comment des choix d'activités pédagogiques et d'exploitation de ressources culturelles permettent aux étudiants de mieux comprendre la francophonie canadienne et leur donnent la confiance d'y participer. Le premier cours, FLS 4775, traite du développement des compétences interculturelles (Byram, 2003). Il présente des projets pédagogiques permettant aux étudiants de participer à la francophonie canadienne de manière autonome et éclairée. Le deuxième cours, FLS 4774, accorde une valeur didactique à la compréhension des variétés locales de la langue pour mieux conscientiser les étudiants à la fonction symbolique de celle-ci (Cuq et Gruca, 2002) et les amener à communiquer avec les locuteurs natifs de manière plus autonome.

\section{Le développement des compétences interculturelles}

\section{Sensibiliser et accompagner}

En tant que passeur interculturel, l'enseignant de FLS accompagne les apprenants dans leur découverte et leur appropriation de la culture francophone (Thibault, 2014). Ce cheminement se fait à travers un apprentissage qui combine la théorie et l'expérience, c'est-à-dire le «sensé » et le «senti » (Gohier, 2002, p. 231). Une telle démarche entraîne une réflexion personnelle et collective sur l'Autre et le Moi, et crée un effet de distanciation qui contribue au déve- 
loppement non seulement des compétences interculturelles, mais aussi de la construction identitaire des apprenants (Byram, 2003). Ainsi, l'apprentissage de la langue va de pair avec l'apprentissage du décodage des signes culturels qui s'y rattachent. Ce double apprentissage est l'objet principal du cours qui sert de contexte à la réflexion de pratique présentée dans cette section.

\section{Cours Culture et communication en FLS}

Les trois grands objectifs du cours suivent la progression suivante : identifier et comprendre la francophonie canadienne, notamment la francophonie minoritaire en Ontario, et y participer. L'apprentissage de la langue/culture se compose de cours magistraux et de travaux de réflexion et de pratique, individuels et collectifs. Tout en poursuivant le perfectionnement de leurs compétences linguistiques, les étudiants observent, analysent et commentent les liens entre langue et culture dans la société canadienne et dans leur propre vie. Ainsi, le sensé et le senti demeurent présents tout au long du cours. De plus, les travaux présentés en classe sont systématiquement suivis de discussions et certains travaux écrits sont également discutés en classe, ce qui permet aux étudiants de développer leurs compétences interculturelles de manière à la fois personnelle et collective.

\section{Identifier et comprendre}

En plus des lectures d'articles scientifiques qui donnent un contexte théorique, les étudiants approfondissent leur réflexion lorsqu'ils rédigent une autobiographie langagière, c'est-à-dire un texte de réflexion personnelle qui les amène à exprimer de manière consciente leur rapport au français et à la francophonie, ainsi que leur rapport à l'idée d'identité linguistique, le plus souvent hétérogène et mouvante. Les représentations qui émergent révèlent des questionnements individuels importants qui constituent le point de départ du cours où l'identité francophone et l'identité bilingue, aux niveaux individuel et collectif, occupent un rôle central.

Dans un travail écrit puis présenté en classe sous forme d'affiche, les étudiants analysent également les liens entre la langue et la culture à travers une étude comparative du traitement par les médias anglais et français des événements majeurs au Canada. Les étudiants sont libres de choisir un événement dans une liste de suggestions fournie par la professeure ou dans l'actualité récente. Bien que de nature plutôt théorique, ce travail est parfois en lien direct avec le vécu ou les intérêts personnels des étudiants. Parmi les sujets qui ont attiré plusieurs étudiants figurent le succès du Nouveau Parti démocratique aux élections fédérales de 2011, les grèves étudiantes au Québec, l'abolition du registre des armes à feu, les Jeux olympiques de Vancouver et les référendums sur l'indépendance du Québec. 


\section{Participer}

Une deuxième série de travaux permet aux étudiants d'approfondir, de manière expérientielle, leur connaissance et leur compréhension de la francophonie, notamment locale. Le travail de reportage combine la théorie, l'expérience, une rédaction et une présentation devant la classe. Les étudiants font une recherche et rencontrent des acteurs de la francophonie comme des artistes (Michel Ouellette, Jean-Marc Dalpé, Chantal Lavallée) ou des membres clé d'une institution culturelle de leur choix (le Centre national des arts (CNA), l'Association canadienne-français de l'Ontario, la Fédération de la jeunesse francoontarienne, le Patro d'Ottawa, la Fédération culturelle canadienne-française). Les étudiants sont sensibles à la passion et à la fierté des personnes qu'ils rencontrent. Ils font l'expérience de la vitalité culturelle de la francophonie qui les entoure et à laquelle ils se sentent parfois invités à participer, comme dans le cas du Bal de neige d'Ottawa, du Mois de la francophonie ou du Festival franco-ontarien qui a lieu au mois de juin.

Les étudiants qui souhaitent participer activement à la francophonie dans le cadre du cours choisissent plutôt l'option d'apprentissage par l'engagement communautaire (AEC), qui comprend 30 heures de bénévolat (dans un journal étudiant, un hôpital, une école française ou un programme d'immersion), une rédaction et une présentation en classe. Cette formule connaît un succès grandissant chez les aspirants enseignants, qui sont de plus en plus nombreux à proposer eux-mêmes l'école de leur choix (comme l'ont fait trois des cinq étudiants ayant choisi l'option AEC pendant la session d'hiver 2014). En classe, ces étudiants expliquent les bénéfices linguistiques, pratiques et culturels d'une telle expérience. Ce sont eux qui expriment avec le plus de confiance leur sentiment d'appartenance à la francophonie.

Finalement, le projet de critique d'un événement culturel qui combine expérience, rédaction critique et discussion en classe procure aux étudiants une occasion exceptionnelle de vivre une expérience d'immersion culturelle en français. Plusieurs pièces de théâtre et spectacles de contes et même une émission de télévision enregistrée sur le campus leur sont suggérés. En cours, ils ont appris l'importance du thêâtre et de la poésie dans l'identité franco-ontarienne grâce à la lecture à voix haute d'extraits de textes fondateurs comme Le chien (Dalpé, 2003) et L'homme invisible/The Invisible Man (Desbiens, 2008). Ils sont donc conscients de la complicité présente entre les artistes et le public dans des lieux comme la Quatrième salle du CNA — où se produit la série des Contes nomades - le studio et le théâtre du CNA — où se produit le théâtre en français - ou encore le Théâtre de l'île à Gatineau où certains étudiants ont vu À toi pour toujours, ta Mari-Lou en 2014 (Tremblay, 1971). La discussion en classe permet aux étudiants de partager leurs impressions et d'identifier non seulement les défis mais aussi la fierté de participer à un événement culturel 
authentique. Qu'ils aient aimé ou pas le spectacle, ils reconnaissent l'importance de vivre personnellement la culture francophone pour la comprendre.

Un sondage administré en classe à la fin de la session d'hiver 2014 demandait aux étudiants d'indiquer sur une échelle de 0 (pas pertinent du tout) à 5 (tout à fait pertinent) la pertinence de chacun des travaux pour leur apprentissage et leur compréhension de la culture francophone canadienne. Ce sont les travaux de nature expérientielle comme «le reportage ou l'AEC » (moyenne de $4,75 / 5)$ et le travail de critique d'événement culturel $(4,45 / 5)$ qui sont arrivés en tête. Deux commentaires résument bien cette tendance : « Le reportage et l'événement culturel étaient les deux meilleurs parce que ça nous forçent d'aller hors du classe pour l'information et d'immerser dans la culture français » et «J'aime bien que nous avons besoin d'aller participer dans la communauté franco-ontarien à travers l'AEC et l'événement culturel. »

Le cours Culture et communication en FLS développe la littératie culturelle des étudiants. Ils apprennent à «lire» le paysage culturel et, pour certains, développent un sentiment de complicité, voire d'appartenance, quand ils repèrent un drapeau vert et blanc arboré d'un trillium et d'une fleur de lys, passent devant La Nouvelle Scène ou l'Hôpital Montfort, ramassent le journal étudiant La Rotonde sur le campus de l'Université, ou entendent un artiste francophone à la radio. La dimension humaine et concrète des travaux pratiques sensibilise les étudiants et les outille à comprendre une francophonie que certains d'entre eux pourront promouvoir auprès de leurs propres élèves. Il serait donc profitable que tous les futurs enseignants de FLS suivent un cours de sensibilisation culturelle comparable.

\section{Le développement de la compétence sociolinguistique}

Dans cette deuxième partie, il sera question du développement de la compétence sociolinguistique chez les apprenants avancés de FLS dans le cours Compréhension du français d'ici. Dans un premier temps, à partir des descriptifs du CECRL et des Niveaux de compétence linguistique canadien (NCLC), nous décrirons ce qu'est la compétence sociolinguistique et présenterons les savoirs linguistiques attendus chez les apprenants de niveau avancé en ce qui a trait au développement de cette compétence. Dans un deuxième temps, nous décrirons comment le professeur de langue, à travers une démarche pédagogique structurée, conscientise les étudiants à la dimension sociolinguistique de la langue et favorise ainsi la compréhension de la variété du français parlé au Canada.

\section{La compétence sociolinguistique}

La compétence sociolinguistique « régit l'incidence de l'environnement sur l'usage de la langue »(Canada, CIC, 2012, p. 8), et elle porte sur le développement et l'acquisition de savoirs linguistiques et de savoir-faire qui permet- 
tront à l'apprenant de «faire fonctionner la langue dans sa dimension sociale » (Conseil de l'Europe, 2005, p. 23). Ainsi, nous pouvons affirmer que la compétence sociolinguistique, tout comme la compétence interculturelle, est une compétence indispensable pour participer activement à la communauté francophone canadienne et interagir avec elle.

Bien que cette compétence fasse partie de la majorité des curriculums des programmes de formation linguistique primaires, secondaires et postsecondaires, l'importance qu'on y accorde semble être très variable. Pourtant, «la composante socio-linguistique (très proche de la compétence socio-culturelle) est à prendre en compte car la langue, dans ce qu'on en fait, est un phénomène social. Parler n'est pas uniquement faire des phrases. Entrent en jeu, ici, des traits relatifs à l'usage de la langue ...» (Éduscol, 2013, section 4.2).

Les savoirs attendus relatifs au développement de cette compétence chez les apprenants de niveau $\mathrm{C}^{1}{ }^{1}$ du CECRL, niveau avancé correspondant à la majorité des étudiants inscrits à ce cours, sont scindés en cinq catégories, toutes étroitement liées à l'usage de la langue dans sa dimension sociale :

- les marqueurs de relations sociales;

- les règles de politesse;

- les expressions populaires ;

- les différences de registre ;

- les dialectes et accents (variations phonétiques, grammaticales et lexicales).

En ce qui a trait aux NCLC, les savoirs attendus en compréhension de l'oral pour la compétence sociolinguistique chez les apprenants de niveau $9^{2}$, niveau seuil des niveaux avancés, sont définis sous forme d'objectifs spécifiques :

- comprend un langage imagé;

- reconnaît les nuances dans différents styles (formels et informels), registres de langue et variétés de la langue;

- reconnait un grand nombre de références culturelles ;

\footnotetext{
${ }^{1}$ Niveau $\mathrm{C} 1$ du CECRL, intitulé «niveau autonome»: «Ce niveau semble être caractérisé par le bon accès à une large gamme de discours qui permet une communication aisée et spontanée ...» (www.delfdalf.fr/niveau-c1-du-cecr-cadre-europeencommun-de-reference-pour-les-langues. html).

${ }^{2}$ Niveau 9, niveau seuil du stade 3 des NCLC : «À ce dernier stade, les apprenants ont une conception précise du but de la communication et du public auquel ils s'adressent (y compris le niveau de familiarité avec l'interlocuteur, les formules de politesse et les formalités, le registre et le style appropriés ...)» (Canada, CIC, 2012, p. 9).
} 
- peut parfois avoir de la difficulté à comprendre certaines manifestations d'humour et certaines expressions idiomatiques peu fréquentes ;

- saisit, par inférence, le parti pris de l'interlocuteur, son attitude, son intention ainsi que des informations sur son bagage culturel;

- repère des indices permettant de demander la collaboration, d'interrompre ou de s'imposer poliment;

- peut comprendre certains accents régionaux et des régionalismes.

Comme on peut le voir, les savoirs correspondant à la compétence sociolinguistique touchent tous les registres de langue. Toutefois, tel que mentionné au premier paragraphe de cette section, dans le cadre du cours dont il est question dans cet article, nous nous sommes limitées aux différents paramètres sociolinguistiques de la langue parlée au Canada et plus particulièrement aux registres courant et familier.

\section{Rôle et démarche pédagogique : cours Compréhension du français d'ici}

Pour mieux connaître la perception des étudiants sur la langue parlée dans le contexte canadien et mieux orienter notre enseignement en fonction des besoins des étudiants, nous avons développé un questionnaire anonyme et facultatif afin de recueillir des informations au début et à la fin du cours. Les questions de ce questionnaire portaient sur différentes perspectives du registre familier du français : perception, degrés de compréhension, besoin de formation.

Les réponses recueillies nous ont permis de prendre davantage conscience de la perception des étudiants quant à leur compétence linguistique et de constater que la langue parlée, la langue familière dans le contexte canadien, représente un véritable défi, même chez les apprenants avancés. Pour certains, ce défi se traduit par une grande insécurité linguistique lors d'interactions avec les locuteurs natifs en dehors du cadre institutionnel. Les informations recueillies par l'entremise du questionnaire distribué au début du cours ont été déterminantes puisqu'elles ont, tout au long du cours, grandement influencé nos choix pédagogiques.

À titre d'exemple, voici un extrait des réponses recueillies à la question : «Est-ce que la langue parlée, la langue familière, représente un défi pour vous?»

« J'ai des difficultés à comprendre la langue familière parce que je manque

d'expériences dans des situations authentiques. »

«Mon défi c'est la compréhension dehors de l'université, dans des situations sociales. »

«Oui! J'ai l'impression de parler comme un textbook. Ce n'est pas la langue de tous les jours. » 
«Je n'ai pas l'habitude de parler français en dehors du cours et je n'ai jamais appris la langue familière. »

«C'est difficile de prendre part à des conversations et se sentir francophone. »

« J'ai des amis francophones, grâce eux je comprends mieux la langue familière. »

«Comme je n'entends pas souvent la langue familière, j'ai des difficultés à comprendre les accents et les expressions. »

En tant que professeures de langue, nous nous sommes donné comme objectif pédagogique pour ce cours de conscientiser les étudiants à la fonction symbolique de la langue, « ... celle qui sert aux partenaires de la situation communicative à laisser des traces de leur être et de leurs appartenances » (Cuq et Gruca, 2002, p. 85). Ainsi, nous souhaitons amener les étudiants à mieux comprendre non seulement la langue, mais aussi le concept de variété de langue. Il nous apparaît important à ce stade de l'apprentissage de comprendre ce qui varie dans la langue, pourquoi elle varie, et quels facteurs sociaux, géographiques, historiques et culturels contribuent à l'évolution du français dans le contexte canadien et donnent au français canadien sa « couleur locale ». Notre but est donc d'amener les étudiants à mieux comprendre ce qui est sous-jacent à la variété de langue qui les entoure afin de ne pas reléguer systématiquement à l'erreur les variations linguistiques canadiennes. «Avec le temps, les apprenants entreront en contact avec des locuteurs d'origines variées. Avant d'adopter pour eux-mêmes des formes dialectales, ils doivent prendre conscience de leurs connotations sociales et de la nécessité d'être cohérent et consistant » (Conseil de l'Europe, 2005, p. 95).

Dans sa finalité, cette démarche de conscientisation et de compréhension vise à encourager les étudiants et à leur donner la confiance nécessaire pour interagir avec les locuteurs natifs de manière plus éclairée et plus autonome. Une démarche pédagogique en adéquation avec l'objectif général du cours : sensibiliser l'étudiant au français parlé du Canada français, surtout aux niveaux familier et populaire, dans ses composantes phonétique, morphosyntaxique, lexicale et socioculturelle.

Scindé en deux parties, le cours se déroule dans un premier temps en salle de classe, puis en laboratoire de langue. Les notions à l'étude visent la compréhension de concepts clés de la sociolinguistique : variété de langue, communauté linguistique, registre de langue, régionalismes, calques, emprunts, néologismes. Ces concepts sont mis en lumière à partir de différents corpus de variations phonétiques, grammaticales et lexicales (emprunts, calques, créations lexicales, jurons) de fréquence élevée et provenant d'enregistrements variés avec des locuteurs natifs du Québec, de l'Ontario et de la péninsule acadienne.

Les activités d'apprentissage proposées sont du type : repérage et discrimination de variations linguistiques; écoute de dialogues en français familier; 
transcription en français standard; écoute d'émissions, de séries télévisées ou d'extraits de films canadiens-français récents ; écoute d'entrevues en langue familière; lecture de bandes dessinées canadiennes-françaises; sortie au théâtre (pièce du répertoire québécois ou franco-ontarien); et écoute de chansons de groupes ou chanteurs canadiens-français.

Toutes ces activités d'écoute, qu'elles soient formatives ou sommatives, sont accompagnées de questions portant sur l'étude de la langue. Le dictionnaire du logiciel Antidote et le dictionnaire $U_{S I T O}{ }^{3}$ servent d'outils de référence pour documenter le travail des étudiants. Les corrections et discussions en classe viennent par la suite renforcer leurs connaissances.

$\mathrm{Au}$ terme du cours, les étudiants ont de nouveau été invités à répondre au questionnaire anonyme et facultatif. À ce stade, nous voulions davantage connaître, après 12 semaines de formation, leur point de vue quant à leur apprentissage et leur réflexion sur le français parlé au Canada, leur perception de ce registre de langue et, finalement, le développement du sentiment de confiance en L2 lors d'interactions dans des situations authentiques en dehors du campus universitaire. Les réponses à la question suivante nous apparaissent révélatrices : «Selon vous, est-il important d'enseigner la compréhension de la langue parlée, la langue familière, à votre niveau?»

Extrait du corpus des réponses recueillies :

«C'est une connaissance que tous les apprenants de FLS au Canada doivent avoir. »

«Oui, je me sens plus francophone!»

« Je dirais que maintenant je suis généralement capable de différencier les expressions familières et standards. »

«C'est important surtout si on veut enseigner la langue. Il faut comprendre la culture et les régionalismes. »

«Oui, je veux retourner au théâtre à Gatineau, avant je n' avais pas la confiance !»

«La langue familière est importante pour socialiser dans la communauté. »

«Je veux travailler en français et pour ça c'est important de distinguer les registres de langue. »

Les données recueillies, comme le démontrent les extraits des réponses aux questions du questionnaire présentées dans cet article, abondent dans le même sens et nous permettent donc d'affirmer que notre démarche a porté fruit. Dans une optique pédagogique plus large, il nous apparaît important de réaffirmer la nécessité d'accorder une importance, dans tous les programmes de formation de FLS au Canada, au développement de la compétence sociolinguistique chez nos apprenants de niveau avancé, et ce, parallèlement au développement des autres compétences générales et communicatives langagières.

\footnotetext{
${ }^{3}$ Disponible à : www.usito.com.
} 


\section{Conclusion}

Dans le contexte de l'apprentissage du FLS au niveau avancé, la littératie englobe les compétences socioculturelles et sociolinguistiques. Plus cette littératie est développée, plus l'apprenant atteint un niveau d'autonomie dans sa connaissance et ses compétences vis-à-vis de la culture et des variétés de langue, et dans sa capacité à participer à la francophonie. Les documents de référence comme le CECRL, les NCLC ou encore le curriculum révisé de FLS de l'Ontario formulent des objectifs clairs à ce propos. Toutefois, la recherche démontre que les professeurs de FLS canadiens reconnaissent ne pas avoir atteint le niveau d'autonomie nécessaire pour transmettre efficacement langue et culture (Salvatori et Macfarlane, 2009). Contrairement aux attentes officielles, les enseignants ne sont donc pas en mesure de bien remplir leur rôle de modèle linguistique et culturel. Par ailleurs, les apprenants de niveau avancé qui souhaitent devenir bilingues ou devenir enseignants de FLS remarquent qu'ils manquent d'opportunités de développer une telle littératie au cours de leur formation postsecondaire (Lamoureux et Cohen, 2012; Moor et Lamoureux, 2012).

Pour résorber cet écart entre attentes officielles et réalité, il est pressant de multiplier les cours et les projets mettant les apprenants en contact avec la culture francophone et ses variétés linguistiques. Deux cours au choix enseignés dans le programme de FLS de l'Université d'Ottawa répondent à un tel besoin en combinant théorie et pratique. Le cours Culture et communication en FLS conscientise les étudiants à la réalité de la francophonie canadienne et surtout ontarienne. Les apprenants découvrent la vitalité de communautés en situation de minorité linguistique et apprennent à décoder le paysage culturel francophone. Ils explorent le concept d'identité linguistique, rencontrent des acteurs de la communauté locale et participent à des événements culturels de leur région. Certains font même un stage comme assistant ou assistante de professeur de français dans les écoles. Pour sa part, le cours Compréhension du français d'ici conscientise les étudiants à la dimension symbolique de la langue en les exposant aux variétés du français parlées au Canada. Les questionnaires de début et de fin de session permettent de conclure que la démarche pédagogique établie dans ce cours répond aux besoins des étudiants. Ceuxci apprennent à identifier les variétés linguistiques et les registres de langue, comprennent mieux leur valeur et leur raison d'être et se sentent davantage capables de comprendre les discours venant de différentes régions du Canada. Les activités d'apprentissage en classe, en laboratoire et dans la communauté locale développent la confiance des étudiants et les encouragent à interagir avec les francophones.

Les pratiques d'enseignement décrites dans le présent article illustrent bien comment développer les compétences socioculturelles et sociolinguis- 
tiques des étudiants de FLS de niveau avancé. Il y aurait lieu de systématiser cette démarche dans les programmes de FLS et de formation des maîtres afin d'outiller les futurs enseignants. Par ailleurs, il faudrait multiplier les opportunités de formation et d'autoformation des enseignants déjà en fonction pour qu'il leur soit plus facile d'accompagner les apprenants dans leur découverte de la richesse de la francophonie canadienne.

\section{Références}

Byram, M. 2003. Introduction. Dans M. Byram (dir.), La compétence interculturelle. Strasbourg, Éditions du Conseil de l'Europe.

Conseil de l'Europe. 2001. Un cadre européen commun de référence pour les langues : apprendre, enseigner, évaluer. Paris, Didier. Consulté le 27 août 2014. Disponible à : www.coe.int/t/dg4/linguistic/Source/Framework_FR.pdf.

Conseil de l'Europe, Division des politiques linguistiques. 2005. Le cadre européen commun de référence pour les langues. Paris, Didier.

Cuq, J.-P. et I. Gruca. 2002. Cours de didactique du français langue étrangère et seconde. Grenoble, Presses Universitaires de Grenoble.

Dalpé, J.-M. 2003. Le chien. Sudbury, Éditions Prise de parole. [1987]

Desbiens, P. 2008. L'homme invisible/The Invisible Man. 3e éd. Sudbury, Éditions Prise de parole.

Éduscol. 2013 (mars). Le CECRL : une base européenne pour l'enseignement des langues. Consulté le 6 août 2014. Disponible à : eduscol.education.fr/cid45678/ cadre-europeen-commun-de-reference-cecrl. html.

Gohard-Radenkovic, C. 1995. Compétences culturelles de l'enseignement et de ses publics apprenant la langue à des fins universitaires et/ou professionnelles. Thèse de doctorat, Paris 3.

Gohier, C. 2002. La polyphonie des registres culturels, une question de rapport à la culture : l'enseignant comme passeur, médiateur, lieur. Revue des sciences de l'éducation, 28, pp. 215-236.

Lamoureux, S. et E. Cohen. 2012. Dans l'ombre du Bonhomme Carnaval et de la Tour Eiffel : formation initiale et approche culturelle de l'enseignement du FLS en milieu minoritaire (Ontario). Communication présentée lors du Colloque DILTEC 2012, Langues, cultures, sociétés : interrogations didactiques, Université Paris 3 Sorbonne Nouvelle, Paris.

Moor, B. et S. Lamoureux. 2012. La place de la culture francophone dans l'enseignement du français langue seconde. Affiche présentée à la foire de recherche du Projet d'initiation à la recherche au premier cycle, Université d'Ottawa, mars 2012.

Ontario, Ministère de l'Éducation. 2013a. A framework for French as a second language in Ontario schools : Kindergarten to grade 12. Toronto, Imprimeur de la Reine pour l'Ontario.

Ontario, Ministère de l'Éducation. 2013b. The Ontario Curriculum, revised. French as Second Language. Core French Grades 4-8, Extended French Grades 4-8 and French Immersion Grades 1-8. Toronto, Imprimeur de la Reine pour l'Ontario.

Salvatori, M. et A. Macfarlane. 2009. Profil et cheminement-Appuis pour le dévelop- 
pement des compétences pédagogiques, linguistiques et culturelles des enseignants du FLS. Rapport de recherche. Ottawa, Association canadienne des professeurs de langue seconde (ACPLS).

Thibault, L.V. 2014. La passerelle : comment conscientiser les étudiants de FLS à la culture francophone minoritaire grâce au théâtre. Revue de l'AQEFLS, 31,1, pp. 123-136. Numéro thématique : Culture/interculture : où en sommes-nous?, V. Amireault et Denise Lussier (dir.).

Tremblay, M. 1971. À toi pour toujours, ta Mari-Lou. Montréal, Léméac. 